\title{
Listeria Meningitis and Nontyphi Salmonella Bloodstream Infection in an Infant
}

\author{
Lilly C. Immergluck ${ }^{1,2}$ Trisha Chan ${ }^{1} \quad$ Rebecca Meyer $^{3}$ Saadia Khizer ${ }^{4}$ Van Ngyuen ${ }^{5}$
}

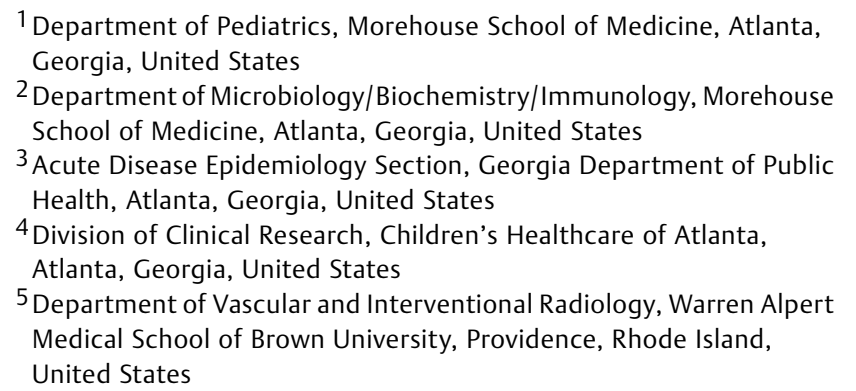

Address for correspondence Lilly Immergluck, MD, Department of Pediatrics, Morehouse School of Medicine, 720 Westview Dr., Atlanta, GA 30310, United States (e-mail: limmergluck@msm.edu).

J Pediatr Infect Dis 2015;10:35-38.

\begin{abstract}
Keywords

- listeria monocytogenes

- nontyphi Salmonella

- infant

To describe a case of an infant with both listeria meningitis and nontyphi Salmonella bacteremia and compare incidence of these two foodborne pathogens in Atlanta, Georgia, United States from 2000 to 2009. An infant presented with two foodborne pathogens without an identifiable source. There are no previous reports of coinfection with L. monocytogenes and nontyphi Salmonella. L. monocytogenes and nontyphi Salmonella remain important foodborne pathogens and source of infection for this age group.
\end{abstract}

\section{Introduction}

Listeria monocytogenes is a foodborne pathogen; invasive infections mainly affect pregnant women, neonates, persons with immune suppression, and the elderly. ${ }^{1}$ The source of infection is frequently linked to ingestion of contaminated food. Salmonella enterica is the most common cause of bacterial foodborne illnesses reported in the United States. ${ }^{2}$ Estimates of the rate of bloodstream infections among children with nontyphi Salmonella gastroenteritis have ranged from 2 to $47 \%^{3-6}$; the risk is greatest for infants less than the age of 2 months. ${ }^{7,8}$ Like listeriosis, patients with immune suppression and the elderly are also at risk of bacteremia in association with salmonellosis. ${ }^{3,9,10}$ We present an infant who had meningitis because of $L$. monocytogenes and concurrently was found to have nontyphi Salmonella bacteremia.

\section{Case Report}

A 2-week-old full-term previously healthy male presented with a history of 4 days of vomiting. The mother denied any

received

March 5, 2015

accepted after revision

April 3, 2015

history of fever, weight loss, difficulty in feeding, rash, diarrhea, or upper respiratory symptoms in her ill infant. The infant's stools were described as loose, yellow-green in color without evidence of blood or mucus. Physical examination revealed an ill appearing but hydrated infant, with a rectal temperature of $39.1^{\circ} \mathrm{C}$, respiratory rate of 60 breaths per minute, pulse of 184 beats per minute, and blood pressure of $87 / 52 \mathrm{~mm} \mathrm{Hg}$. The remainder of his examination was normal.

The mother had no prenatal care before her precipitous spontaneous vaginal delivery of her infant. Review of delivery room medical records revealed no prolonged rupture of membranes and negative maternal screening results for HIV, Hepatitis B, cocaine, opioids, and barbiturates. The infant had a normal physical examination, but because the maternal Group B streptococcus (Streptococcus agalactiae) status was unknown, he was treated with ampicillin and gentamicin until blood cultures taken at birth were negative after 48 hours.

The infant lived at home with his biological mother, ten other children (ages 1 year to 16 years) and three other

Copyright @ 2015 by Georg Thieme Verlag KG, Stuttgart · New York
DOI http://dx.doi.org/ 10.1055/s-0035-1554968. ISSN 1305-7707. 
adults. At the time of admission, the mother occupied a room of the house with her four biological children. There was no family history of sickle cell disease or trait, or immune deficiency. No members of the household had any recent history of diarrhea or upper respiratory infection symptoms. The mother denied any history of illness, either at the time of delivery or around the time of the patient's admission. She did not recall ingestion by herself or her children of any of the foods often associated with $L$. monocytogenes or Salmonella. We specifically inquired about ingestion of most foods listed on the Georgia's Department of Public Health Case Report form for both Listeria and Salmonella, ${ }^{11}$ including processed meats (pepperoni, hot dogs, salami), raw or undercooked poultry or pork, cheese products (fresh mozzarella), foods containing raw egg products, and raw or unpasteurized milk. Infant was fed powdered formula at the time of illness; he did also ingest "ready to feed" liquid formula shortly after birth, but he was never breastfed or provided breast milk. Ready-tofeed formula provides manufactured complete nutritional milk product in ready-to-feed bottles. There were no household pets, and no history of contact with reptiles, live chickens, or other birds.

Because of the infant's fever without a clinically obvious focus of infection, an evaluation for clinical sepsis was initiated and he was commenced empirically on ampicillin, cefotaxime, and acyclovir parenterally after specimens were collected for complete blood count, blood culture, urine culture, and cerebral spinal fluid analysis and culture. The infant tested negatively for anti-HIV antibodies. Cerebral spinal fluid culture grew $L$. monocytogenes after 48 hours of incubation. The blood culture grew S. enterica, which the Georgia state health laboratory serotyped as Salmonella serotype I, $4,{ }^{5} 12: \mathrm{i}:-$ using the Kaufmann-White scheme. ${ }^{12}$ Both the L. monocytogenes and Salmonella serotype I, $4,{ }^{5} 12: \mathrm{i}$ :isolates were found to be susceptible to ampicillin. The L. monocytogenes isolate was identified as "nontypeable" by the Centers for Disease Control and Prevention (CDC) and no serogroup or serovar could be determined; no other L. monocytogenes isolates submitted during this same year had the same pulsed field gel electrophoresis pattern. No further workup was performed on this isolate. The patient was discharged after completing 16 days of parenteral ampicillin and cefotaxime therapy. Both the patient and mother have since then been lost to follow-up.

\section{Discussion}

L. monocytogenes is the infectious agent of listeriosis and usually manifests as meningitis in infants, whereas Salmonella infections typically present as acute enteritis in children. ${ }^{13}$ Both pathogens cause disease after ingestion of contaminated food and there is crossover in the types of food products which may carry both pathogens, for example, dairy products, uncooked fruits, and vegetables. We hypothesize that our infant most likely contracted the two pathogens from his mother or a household contact. We consider that the index case(s) likely acquired both pathogens from the same food source. Given that neither the mother nor any hospital contact reported recent illness it is possible that the source person may have been an asymptomatic carrier. Another possibility is related to the ability of $L$. monocytogenes to form biofilms and survive in conditions which many other pathogens cannot. ${ }^{14}$ In this case, it is also possible that the infant's feeding supplies, for example, bottle, nipples, pacifiers were contaminated.

Although this infant had late-onset listeriosis (infection after 7 days of life), ${ }^{15}$ it is possible the infant acquired both foodborne pathogens perinatally or in utero, and since he received ampicillin at the time of birth, the symptoms of bacterial infection could have been delayed because of this "pretreatment."

The infant presented was more susceptible to coinfection primarily because of his young age; newborn infants' immune function is not completely mature and thus these babies are essentially immune compromised. These two infections when they occur in the neonatal population require hospitalization, adding to the cost of health care. Thus, from a public health perspective, it is vital to prevent these infections from

Frequency of Pediatric Listeria monocytogenes and Salmonella enterica Infections, by Age Group, in Georgia (2000-2009)

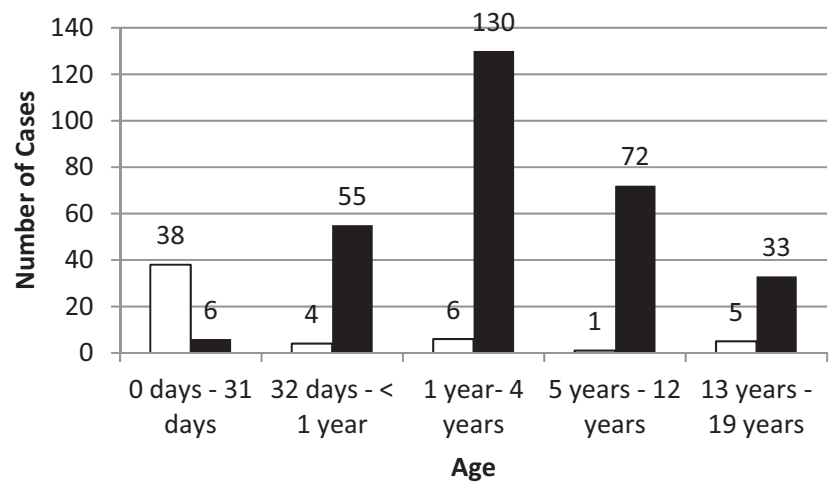

$\square$ Listeria monocytogenes

- Salmonella serotype I 4 [5],12:i:-

Fig. 1 Number of pediatric cases (< 19 years) during the 10 years (2000-2009) for Listeria monocytogenes and Salmonella enterica infections for the state of Georgia. 


\section{Listeria monocytogenes Prevalence, 2000- 2009}
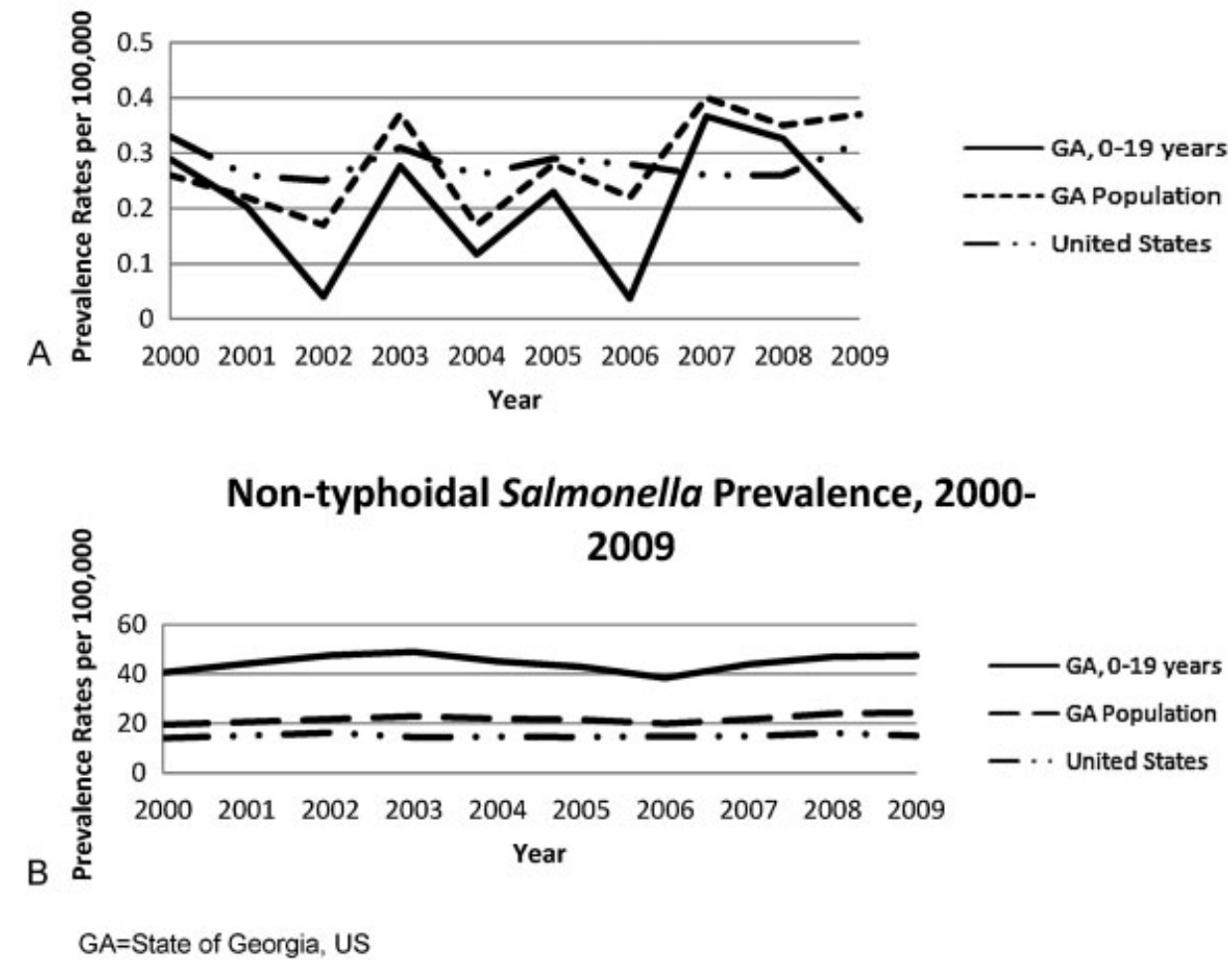

Fig. 2 Comparison of the prevalence (number of cases per 100,000 of population category) of Listeria monocytogenes (Panel A) and nontyphoidal Salmonella (Panel B) among the Georgia pediatric population (birth to $<19$ years), overall Georgia population, and United States population. GA, State of Georgia, United States.

occurring primarily by educating the public on what are the most common food sources for these pathogens and how to prevent ingestion of such contaminated foods, particularly among at risk populations, for example, pregnant women or elderly.

In Georgia, pediatric cases of listeriosis occur predominantly in infants younger than 1 month and although the frequency of Salmonella cases were highest among children of the age of 1 to 4 years, the highest rate was seen in those infants younger than 1 year (-Fig. 1) (R. Meyer, MPH, personal communication, October 1,2014$).^{16}$ During the 10 -year period (2000-2009), the incidence of $L$. monocytogenes infections ranged from 0.17 per 100,000 persons to 0.40 per 100,000 persons. (Meyer, personal communication, October 1, 2014). There were a total of 54 cases of listeriosis in those younger than 19 years during this 10 -year period. Of the 54 cases, 12 had meningitis and 5 had bloodstream infections. Georgia's listeriosis prevalence is comparable to the United States ( - Fig. 2, Panel A). Several listeriosis clusters were noted during this 10-year span; however, many were multistate clusters involving very few patients with Georgia case. The increase during these years is most likely because of the fluctuation in yearly incidence seen in many foodborne illnesses.

We were unable to determine the source(s) for coinfection of our case. There were no outbreaks of $L$. monocytogenes or nontyphi Salmonella species (including Salmonella serotype I $4,{ }^{5} 12: \mathrm{i}$ :- in the area or immediate surrounding areas where this patient resided in Georgia. The incidence of nontyphi Salmonella in Georgia ranged from 19.51 to 24.31 cases per 100,000 persons, which is comparable to what has been reported nationally (Meyer, personal communication, October 1,2014$).{ }^{16}$ The average rate in those younger than 19 years with nontyphi Salmonella in Georgia was 44.6 cases per 100,000 persons and 1.13 cases per 100,000 persons for Salmonella serotype I $4,{ }^{5} 12$ :i:- (-Fig. 2, panel B) (R. Meyer, $\mathrm{MPH}$, personal communication, October 1, 2014).The specific serovar identified in our patient accounts for only $3 \%$ of reported Salmonella infections within the catchment area surveyed by the Foodborne Disease Active Surveillance Network (FoodNet) of the CDC's Emerging Infections Program. ${ }^{17}$

Environmental contamination could have been a possible source of infection; however, environmental contamination commonly seen in southeastern United States, such as, waterborne or amphibian contact did not seem to be the cause in our case. ${ }^{18,19}$

Our case report is an example of the invasiveness associated with these two foodborne pathogens and public health awareness and prevention should target those populations who are most vulnerable to invasive disease.

Funding

This project was supported in part by funds received from the Clinical Research Education and Career Development 
(CRECD) Grant number 8R25MD007589-10 and the National Center for Advancing Translational Sciences of the National Institutes of Health under Award Number UL1TR000454. The content is solely the responsibility of the author and does not necessarily represent the official views of the National Institutes of Health.

\section{Acknowledgments}

We thank Melissa Tobin-D’Angelo, MD, MPH for her review and comments and Mr. Kevin Thornton, BS for his review and edits.

\section{References}

1 Baltimore R. Listeria monocytogenes. In: Kliegman R, Stanton B, Shor N, Behrman R, St Geme J, eds. Nelson Textbook of Pediatrics. Philadelphia, PA: Elsevier Saunders; 2011:929

2 Scallan E, Hoekstra RM, Angulo FJ, et al. Foodborne illness acquired in the United States-major pathogens. Emerg Infect Dis 2011; 17(1):7-15

3 Hyams JS, Durbin WA, Grand RJ, Goldmann DA. Salmonella bacteremia in the first year of life. J Pediatr 1980;96(1):57-59

4 Rani H, Singla N, Chander J, Jain S. Infant bacteremia due to Salmonella typhimurium. Indian J Pathol Microbiol 2011;54(2):417-418

5 Shkalim V, Amir A, Samra Z, Amir J. Characteristics of non-typhi Salmonella gastroenteritis associated with bacteremia in infants and young children. Infection 2012;40(3):285-289

6 Bar-Meir M, Raveh D, Yinnon AM, Benenson S, Rudensky B, Schlesinger Y. Non-Typhi Salmonella gastroenteritis in children presenting to the emergency department: characteristics of patients with associated bacteraemia. Clin Microbiol Infect 2005;11(8):651-655

7 Dhanoa A, Fatt QK. Non-typhoidal Salmonella bacteraemia: epidemiology, clinical characteristics and its' association with severe immunosuppression. Ann Clin Microbiol Antimicrob 2009;8:15
8 Huang IF, Kao CH, Lee WY, et al. Clinical manifestations of nontyphoid salmonellosis in children younger than 2 years oldexperiences of a tertiary hospital in southern Taiwan. Pediatr Neonatol 2012;53(3):193-198

9 Workman MR, Philpott-Howard JN, Casewell MW, Bellingham AJ. Salmonella bacteraemia in sickle cell disease at King's College Hospital: 1976-1991. J Hosp Infect 1994;27(3):195-199

10 Schutze GE, Schutze SE, Kirby RS. Extraintestinal salmonellosis in a children's hospital. Pediatr Infect Dis J 1997;16(5):482-485

11 Hackbarth A. Foodborne Illness Investigation and Control Reference Manual. Georgia Department of Human Resources-Division of Public Health, Environmental Health Office and Notifiable Disease Epidemiology Section. Atlanta, Georgia: Department of Public Health; 2005:1-15

12 Molbak K, Olsen JE, Wegener HC. Salmonella infections. Chapter 3. In: Riemann HP, Cliver DO, eds. Foodborne Infections and Intoxications. Amsterdam: Elsevier Academic Press (imprint of Elsevier); 2006:57-136

13 Bhutta Z. Salmonella. In: Kliegman R, Stanton B, Shor N, Behrman R, St Geme J, eds. Nelson Textbook of Pediatrics. Philadelphia, PA: Elsevier Saunders; 2011:948-958

14 Renier S, Hébraud M, Desvaux M. Molecular biology of surface colonization by Listeria monocytogenes: an additional facet of an opportunistic gram-positive foodborne pathogen. Environ Microbiol 2011;13(4):835-850

15 Jackson KA, Iwamoto M, Swerdlow D. Pregnancy-associated listeriosis. Epidemiol Infect 2010;138(10):1503-1509

16 Georgia Department of Public Health OASIS Online Analytical Statistical Information System, c2003-2013. Available at: http:// oasis.state.ga.us/oasis. Accessed October 1, 2013

17 Foodborne Diseases Active Surveillance Network. FoodNet Surveillance Report for 2009 (Final Report). U.S. Department of Health and Human Services, Centers for Disease Control; 2009

18 Srikantiah P, Lay JC, Hand S, et al. Salmonella enterica serotype Javiana infections associated with amphibian contact, Mississippi, 2001. Epidemiol Infect 2004;132(2):273-281

19 Clarkson LS, Tobin-D'Angelo M, Shuler C, Hanna S, Benson J, Voetsch AC. Sporadic Salmonella enterica serotype Javiana infections in Georgia and Tennessee: a hypothesis-generating study. Epidemiol Infect 2010;138(3):340-346 